\title{
Fragmentation of tRNA in Phytophthora infestans asexual life cycle stages and during host plant infection
}

\author{
Anna KM Åsman ${ }^{1 * \dagger}$, Ramesh R Vetukuri ${ }^{1 \dagger}$, Sultana N Jahan ${ }^{1}$, Johan Fogelqvist ${ }^{1}$, Pádraic Corcoran ${ }^{1,3}$, \\ Anna O Avrova ${ }^{2}$, Stephen C Whisson ${ }^{2}$ and Christina Dixelius ${ }^{1}$
}

\begin{abstract}
Background: The oomycete Phytophthora infestans possesses active RNA silencing pathways, which presumably enable this plant pathogen to control the large numbers of transposable elements present in its $240 \mathrm{Mb}$ genome. Small RNAs (sRNAs), central molecules in RNA silencing, are known to also play key roles in this organism, notably in regulation of critical effector genes needed for infection of its potato host.

Results: To identify additional classes of sRNAs in oomycetes, we mapped deep sequencing reads to transfer RNAs (tRNAs) thereby revealing the presence of 19-40 nt tRNA-derived RNA fragments (tRFs). Northern blot analysis identified abundant tRFs corresponding to half tRNA molecules. Some tRFs accumulated differentially during infection, as seen by examining sRNAs sequenced from $P$. infestans-potato interaction libraries. The putative connection between tRF biogenesis and the canonical RNA silencing pathways was investigated by employing hairpin RNA-mediated RNAi to silence the genes encoding P. infestans Argonaute (PiAgo) and Dicer (PiDcl) endoribonucleases. By sRNA sequencing we show that tRF accumulation is PiDcl1-independent, while Northern hybridizations detected reduced levels of specific tRNA-derived species in the PiAgo 1 knockdown line.
\end{abstract}

Conclusions: Our findings extend the sRNA diversity in oomycetes to include fragments derived from non-protein-coding RNA transcripts and identify tRFs with elevated levels during infection of potato by $P$. infestans.

Keywords: Argonaute, Dicer, Phytophthora infestans, Potato, RNA silencing, Small RNA, tRF, tRNA

\section{Background}

The mechanisms behind eukaryotic gene regulation have been extensively studied in animal, plant and fungal model organisms. Comparatively less is known about regulation of gene expression in heterokonts (stramenopiles), the eukaryotic group formed by diatoms, brown algae and oomycetes [1]. Members of the latter group resemble fungi in their morphology and lifestyle, but are phylogenetically only distantly related to true fungi $[2,3]$. The oomycetes encompass species living as saprophytes and pathogens of plants, insects, crustaceans, fish, and animals $[4,5]$. The most studied plant pathogenic oomycete is the

\footnotetext{
* Correspondence: Anna.Asman@slu.se

${ }^{\dagger}$ Equal contributors

'Department of Plant Biology, Uppsala BioCenter, Linnéan Centre for Plant Biology, Swedish University of Agricultural Sciences, PO. Box 7080, SE-75007 Uppsala, Sweden

Full list of author information is available at the end of the article
}

potato late blight agent, Phytophthora infestans [6]. The $P$. infestans genome $(\sim 240 \mathrm{Mb})$ is one of the largest known within the genus Phytophthora, the majority comprising repetitive DNA [7]. The genome shows a bimodal organization pattern with densely packed core gene regions, interrupted by repeat-rich regions that are sparsely populated by genes [8]. A closer examination of the repeat-rich regions reveals enrichment for genes encoding diseasepromoting effector proteins, which are at the forefront of evolution in this pathogen $[9,10]$.

RNA silencing encompasses a set of mechanisms present in eukaryotes in which small RNAs (sRNAs) play central roles. It is the vanguard of genome defense against invasive nucleic acids such as transposons, viruses and transgenes $[11,12]$. Double-stranded RNA (dsRNA) from these myriad sources acts as triggers for gene silencing, initiating the degradation of complementary mRNA. This occurs via the 
generation of sRNAs by the class III ribonuclease (RNase) Dicer (Dcr; or Dicer-like, Dcl) and the association of the sRNA with Argonaute (Ago) family proteins. From the plethora of sRNA classes discovered and described, microRNAs (miRNAs), small interfering RNAs (siRNAs), and the Dcl-independent Piwi-interacting RNAs (piRNAs), are the most well characterized subtypes [12,13]. A class of sRNAs that was recently discovered through a number of deep sequencing studies is tRNA-derived RNA fragments (tRFs), 18-46 nt pieces derived from mature tRNA or the $3^{\prime}$ end of precursor-tRNA (pre-tRNA) [14-16]. tRNA fragmentation as a source of sRNA has been documented in organisms from all three domains of life [17].

Over the past decade, several compelling studies have shown that $P$. infestans possesses typical eukaryotic gene silencing pathways [18-22]. Silencing in $P$. infestans is reported to be functional at both the post-transcriptional and the transcriptional level, with sRNAs and heterochromatin formation acting to control transposons and transgenes [23-27]. Transcriptional silencing, and likely heterochromatin formation, has been shown to involve histone modification rather than cytosine methylation, and outward spread of silencing from heterochromatic loci has been detected at distances up to $600 \mathrm{bp}$ [24-27]. Moreover, sRNAs are increasingly recognized as important players in plant-pathogen interactions. A recent report showed that pathogen-to-host sRNA transport resulted in silencing of host immunity genes and promotion of infection [28].

Here, in order to further identify and characterize the sRNA repertoire in $P$. infestans, the reads obtained through deep sequencing of sRNA were mapped to tRNAs. Data from two isolates and four developmental stages showed that the majority of tRFs mapped exclusively to the $5^{\prime}$ half of mature tRNA, had a guanosine at the $5^{\prime}$ end and mapped with the $3^{\prime}$ end in the tRNA anticodon loop region. Analysis of sRNA from infected potato leaves, on the other hand, identified a number of tRFs from $P$. infestans that were most abundant during infection, and the relative proportion of cleavage products from the $5^{\prime}$ and $3^{\prime}$ tRNA halves was found to shift during infection.

\section{Results and discussion}

\section{sRNAs derived from $P$. infestans tRNAs}

sRNA libraries were constructed from mycelia, sporangia, germinating sporangia and germinating cysts of two contrasting isolates (R0 and 3928A) via RNA-adapter mediated ligation. The two isolates differ in their specific virulence phenotypes and mating types: $\mathrm{R} 0$ is of the $\mathrm{A} 1$ mating type and is weakly pathogenic on potato [29], while $3928 \mathrm{~A}$ belongs to the A2 mating type and is highly pathogenic [30]. The proportions of sequences derived from each life cycle stage are listed in Vetukuri et al.
[21]. Here, the total sRNA dataset was mapped to tRNAs (Additional file 1: Table S1), revealing that less than 2\% mapped to tRNA loci (1.9\% in R0 and $1.5 \%$ in 3928A). Based on sequence identity (cut-off $90 \%$ ), all tRNA and tRNA-like sequences from the $P$. infestans genome were sorted into clusters with the cd-hit algorithm [31], generating 230 clusters to which all the sRNA reads were mapped. Previously, deep sequencing from the different life cycle stages reported a skewed distribution of total read numbers [21]. A similar trend was observed for tRFs in this study, where more sRNA sequences mapped to tRNA in the sporangium stage compared to the mycelium stage.

Mapping of sRNAs to individual tRNA clusters revealed that the majority of tRFs were 25-30 nt long and highly enriched for the sense strand, while the overall length distribution peaked at 27 and 30 nt (Figure 1A). Due to the bioinformatics pipeline applied to the SOLiD sequencing data, $30 \mathrm{nt}$ was the upper read length limit.

\section{Most tRFs in $\mathbf{P}$. infestans map to the $\mathbf{5}^{\prime}$ end of mature tRNA}

Studies in humans, plants and protists have revealed tRNA to be cleaved in the open loops of the RNA structure; in the anticodon-loop to generate tRNA halves and in the $\mathrm{D}$ - and T-loops to produce shorter tRFs, referred to as $5^{\prime}$ tRFs and $3^{\prime}$ CCA tRFs $[15,16,32,33]$. A fourth class of tRNA fragments, the $3^{\prime} \mathrm{U}$ tRFs, is produced from the $3^{\prime}$ end of pre-tRNA and ends in the RNA polymerase III termination poly-U tract $[15,16]$. To establish which types of tRNA-derived fragments are present in $P$. infestans, we inspected the proportion of reads aligning to the respective ends of mature tRNA and the $3^{\prime}$ end of pre-tRNA, as well as the read lengths and inferred cleavage sites. Reads mapping with one end within the $5^{\prime}$ or $3^{\prime}$ terminal-most nucleotides of mature tRNA were considered as candidate $5^{\prime}$ or $3^{\prime}$ tRFs, respectively. On average $91 \%$ in $\mathrm{R} 0$ and $89 \%$ in $3928 \mathrm{~A}$ were classified as $5^{\prime}$ tRFs, while only $4 \%$ and $3 \%$ were $3^{\prime}$ tRFs in R0 and 3928A, respectively (Table 1, Additional file 2: Figure S1, Additional file 3: Figure S2). Only three candidate 3' U tRFs were found that started at the first nucleotide downstream of mature tRNA. Two had $3^{\prime} \mathrm{C}$ while one had a single $3^{\prime} \mathrm{U}$, thus none ended in a poly- $\mathrm{U}$ tract. The finding of mostly $5^{\prime}$-mapping tRFs is comparable to the situation in Trypanosoma cruzi, where the overwhelming majority of nutritional stress-induced tRFs originate from the $5^{\prime}$ halves of mature tRNAs [34]. In short, the global profile of $P$. infestans tRFs indicates that these sRNAs originate from specific tRNA cleavage, whereby the $5^{\prime}$ fragments are favored for cellular retention.

\section{Abundant tRFs from tRNA lle_cluster0}

A high number of sRNA reads in the different life cycle stages and isolates mapped to the $5^{\prime}$ part of tRNA Ile_cluster0 (from now on termed as Ile0-5'tRFs). For 


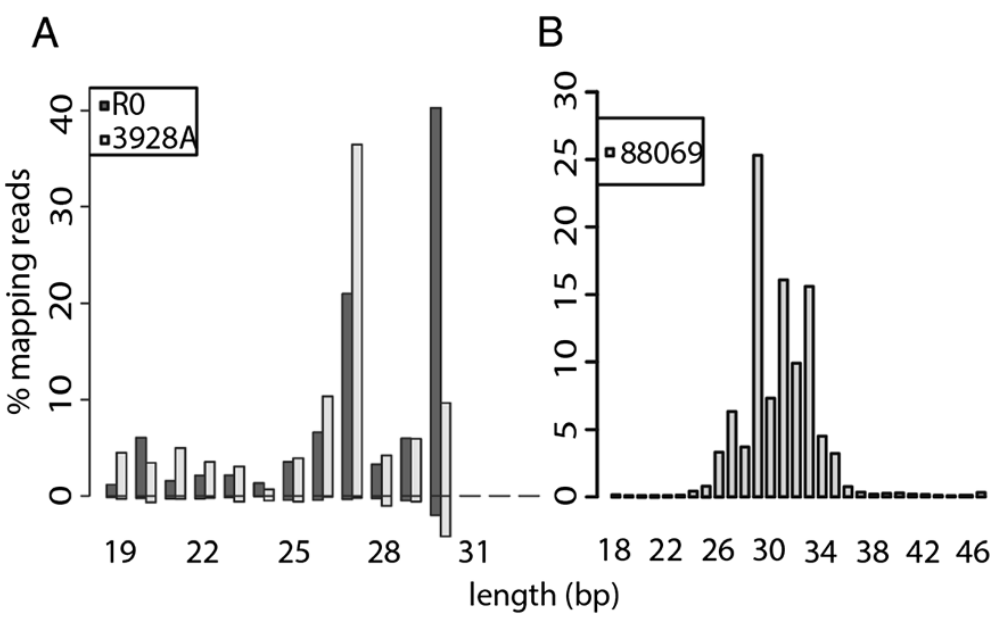

Figure 1 Size distribution of sRNA reads mapping to tRNAs. Shown are total sRNA reads from the mycelium life cycle stage of the three sequenced $P$. infestans isolates. (A) R0 and 3928A. (B) 88069. The percentages of sense and antisense reads are displayed on the positive and negative $y$-axes, respectively.

example, $26 \%$ of the total tRFs identified in the R0 mycelia, and $25 \%$ of total tRFs in the 3928A germinating cysts mapped to this cluster (Additional file 1: Table S1). The number of reads mapping to tRNA Ile_cluster0 (anticodon AAU) was within the top five in all libraries except in the 3928A mycelia, which was also the library having the lowest number of total tRNA-mapping reads (Additional file 1: Table S1). An abundance of tRFs from a few specific tRNAs may suggest isolate- or life cycle specific isoacceptor preference, as was seen in T. cruzi [35]. In this protozoan parasite, tRFs were reported to derive primarily from $\mathrm{tRNA}^{\text {His }}$, $\mathrm{tRNA}^{\mathrm{Arg}}$ and $\mathrm{tRNA}{ }^{\mathrm{Thr}}$, while in the diplomonad parasite Giardia lamblia, the most abundant tRFs during starvation-induced encystment derive from tRNA ${ }^{\text {Asp }}$ and tRNA $^{\text {Gly }}$ [14]. We did not find that the overrepresentation of Ile0-5'tRFs was proportional to the numbers of tRNA ${ }^{\text {Ile }}$ (AAU) genes or the codon frequencies reported from two other Phytophthora species [36].

Table 1 Distribution of tRFs mapping to the $5^{\prime}$ and $3^{\prime}$ halves of mature tRNA in R0 and 3928A

\begin{tabular}{lllllll}
\hline \multirow{2}{*}{ Library } & \multicolumn{3}{l}{ Number of reads } & (\%) \\
\cline { 2 - 5 } & $\mathbf{5}^{\prime}$ & $\mathbf{3}^{\prime}$ & Intermediate & Total & $\mathbf{5}^{\prime}$ \\
\hline R0 germinating cysts & 16130 & 1653 & 604 & 18387 & 88 \\
R0 germinating sporangia & 12669 & 314 & 771 & 13754 & 92 \\
R0 mycelia & 4704 & 118 & 402 & 5224 & 90 \\
R0 sporangia & 31177 & 776 & 1275 & 33228 & 94 \\
3928A germinating cysts & 13054 & 288 & 1021 & 14363 & 91 \\
3928A germinating sporangia & 12992 & 276 & 1015 & 14283 & 91 \\
3928A mycelia & 868 & 69 & 106 & 1043 & 83 \\
3928A sporangia & 10087 & 272 & 675 & 11034 & 91 \\
\hline
\end{tabular}

\section{Characteristics of tRF termini}

Organisms with multiple Ago protein homologs and sRNA classes have mechanisms for pairing of the sRNAs with the correct Ago protein [13]. In plants, this sorting is dictated by the sRNA $5^{\prime}$ terminal nucleotide, such that Arabidopsis thaliana Ago1 recruits mainly miRNAs, of which the majority begin with $5^{\prime} \mathrm{U}$ [37]. P. infestans possesses four distinct Ago genes that are expressed throughout asexual development and during host infection [20]. PiAgo4 and PiAgo5 were shown to positively affect the accumulation of $32 \mathrm{nt}$ long sRNAs from retrotransposons and other mRNAs, while 21 nt sRNAs from similar precursors were associated with PiDcl1 [21]. Nevertheless, any additional roles of the individual Agos in P. infestans gene silencing pathways have not been described. When inspecting the sRNA reads aligning to tRNA in the sequenced life cycle stages, an enrichment of $5^{\prime}$ terminal $\mathrm{G}$ was seen for tRFs of most length classes; on average $66 \%$ of all tRFs from the eight libraries had this particular $5^{\prime}$ nucleotide (Figure 2). $5^{\prime}$ tRFs comprised the majority of our sequencing libraries, thus this observation reflects the strong evolutionary conservation of the tRNA $G_{+1}$ nucleotide, needed for RNaseP cleavage site recognition and successful pre-tRNA processing [38]. tRFs of $27 \mathrm{nt}$ were an exception, since $5^{\prime} \mathrm{U}$ was most prevalent in six out of the eight libraries (the two germinating cyst libraries were the exceptions). This suggests that $27 \mathrm{nt}$ tRFs are generated by a different process and that they might be bound by a distinct PiAgo complex.

To form the mature amino acid-accepting $3^{\prime}$ end, a CCA trinucleotide is added post-transcriptionally to eukaryotic tRNA [39]. While addition of this terminal nucleotide modification is implicated in quality control $[39,40]$, deacylation makes tRNA more prone to CCA loss [32]. We 

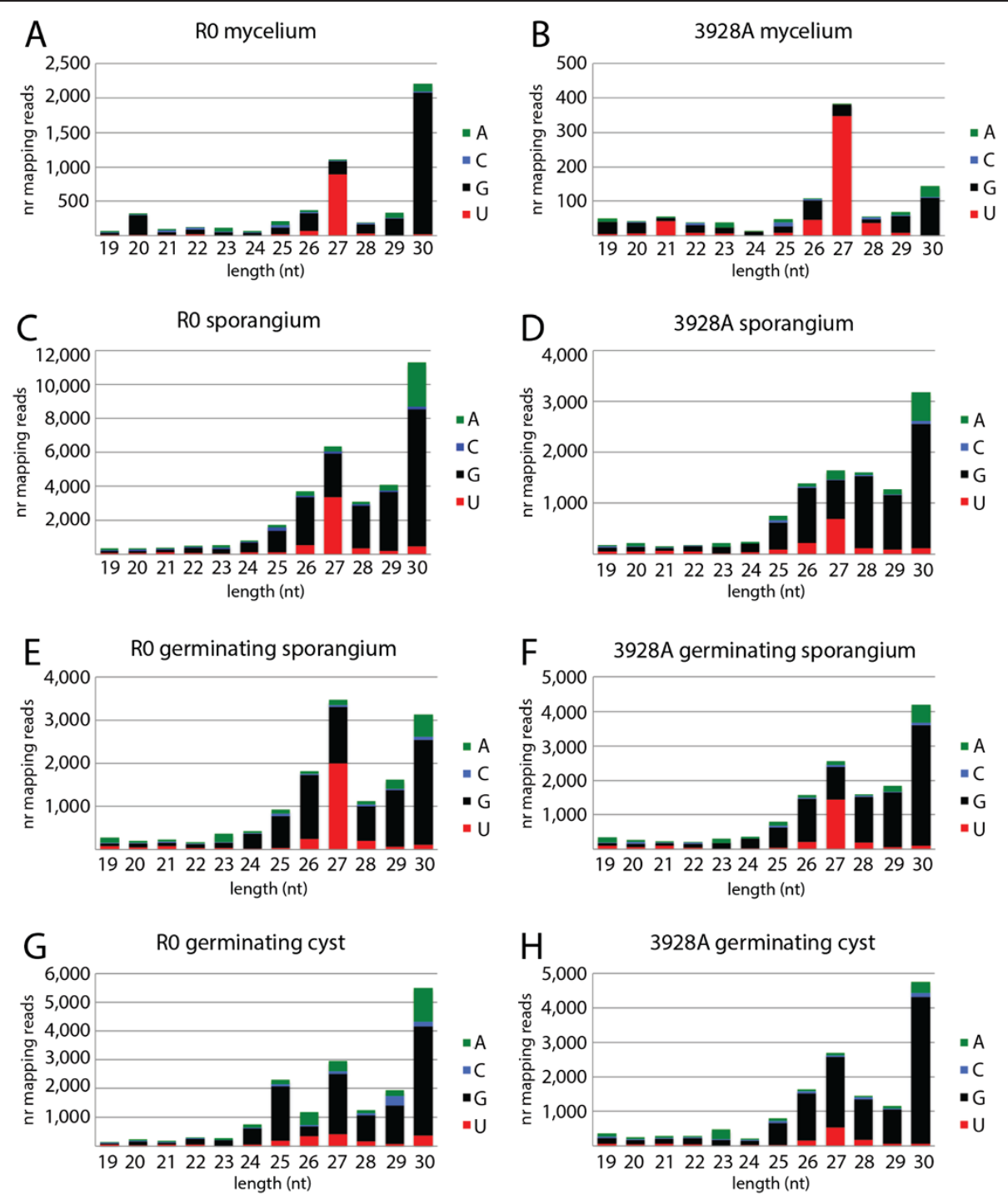

Figure 2 The $\mathbf{5}^{\prime}$ end nucleotide base identity of tRNA-mapping sRNAs in different life cycle stages. (A, C, E, G) Isolate RO. (B, D, F, H) Isolate 3928A. Most tRF size classes started with $5^{\prime} \mathrm{G}$. The $27 \mathrm{nt}$ sRNAs most frequently had $5^{\prime} \mathrm{U}$, except for in germinating cysts.

found very few reads ending in non-template CCA aligning to $P$. infestans $3^{\prime}$ tRFs. The 3' CCA addition takes place before tRNA export from the nucleus and tRFs are cytoplasmic [16,39], so a step in the $P$. infestans $3^{\prime}$ tRF biogenesis pathway likely involves tRNA deacylation.

\section{Experimental validation of tRFs}

To verify the presence of tRNA cleavage products in $P$. infestans, candidate tRFs were chosen for Northern blot analyses of sRNA extracted from sporulating mycelium. First, Ile0-5' tRFs were analyzed. In line with the high number of sRNA sequencing reads from the $5^{\prime}$ fragment of tRNA Ile_cluster0 (Additional file 1: Table S1), strong hybridization signals corresponding to Ile0-5' tRFs were detected (Figure 3A). The length of the most abundant product, 34 nt (tRNA halves), agreed with the SOLiD sequencing data (Figure 4), since $30 \mathrm{nt}$ was the upper read length cut-off. To map the cleavage site positions in the mature tRNA cloverleaf structure, tRNA Ile_cluster0 was in silico folded using Vienna RNAfold [41] (Figure 5). Next, sRNAs from tRNA Thr_cluster1 (Thr1-5' tRFs) were examined. The main tRFs sequenced from this tRNA cluster, at 28 nt long, could be confirmed, although the strongest signal came from half-sized tRNAs (Figures 3A and 4). Both tRNA Ile_cluster0 and tRNA Thr_cluster1 showed strong hybridization signals from $5^{\prime}$ half tRNA molecules. Indeed, fragments of sizes consistent with being tRNA halves were detected for all tRNAs tested by Northern hybridization (Figure 3, Additional file 4: Figure S3, Additional file 5: Figure S4). These results suggest anticodon loop cleavage (at position 32-38) to be common in $P$. infestans, in agreement with observations from 
A
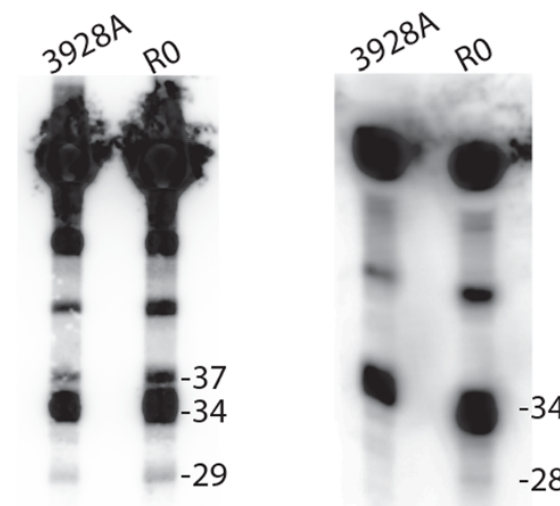

$-28$

Ile0-5'tRF

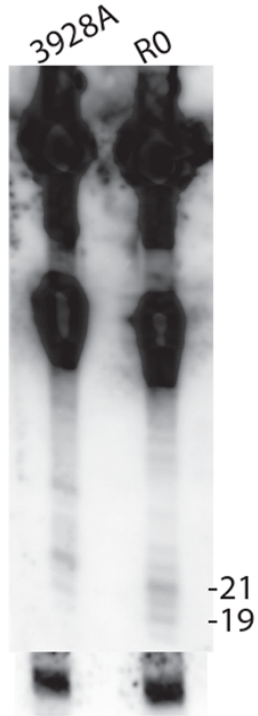

Arg0-tRF

B
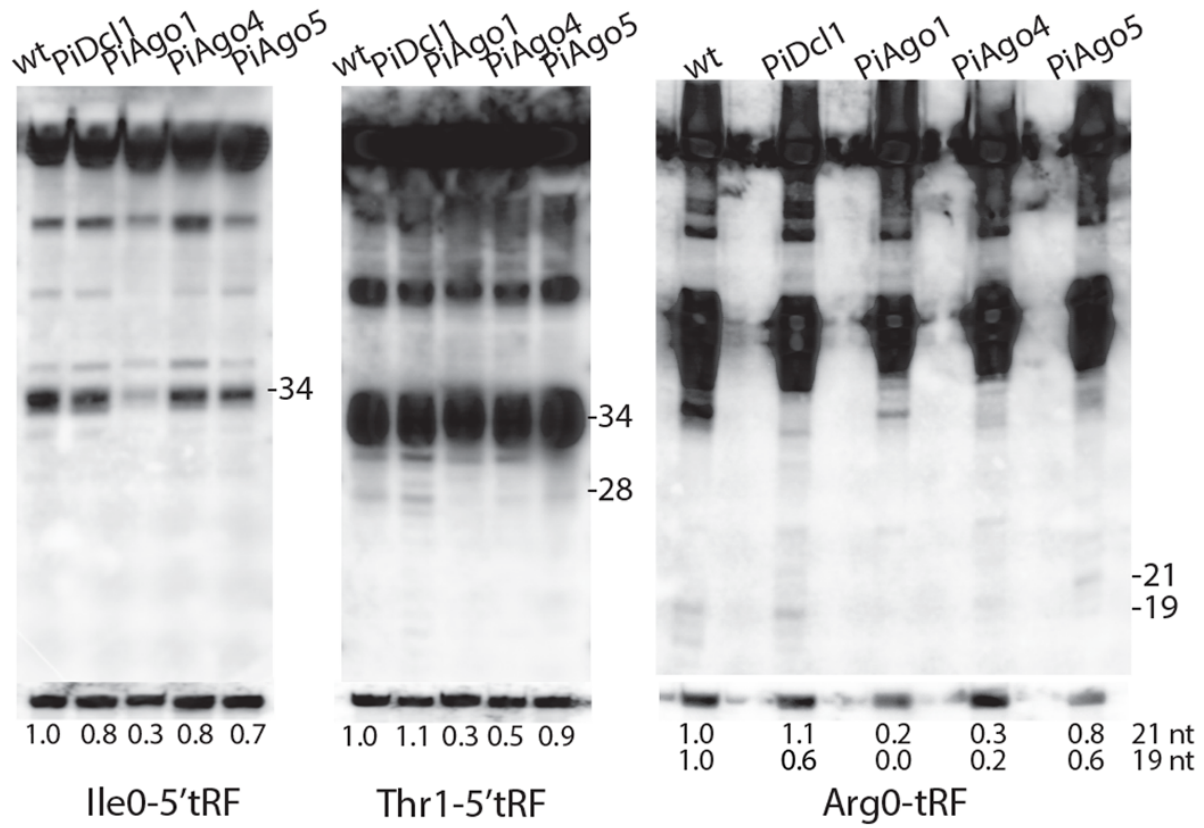

Figure 3 Northern blot detection of sense sRNAs complementary to tRNA. Hybridizations detected sense tRFs from tRNA lle_cluster0, tRNA Thr_cluster1 and tRNA Arg_cluster0 in (A) wild-type (wt) isolates 3928A and R0, and in (B) wt isolate 88069 and transformant lines silenced for PiDCl1, PiAgo 1, PiAgo4, PiAgo5. Approximate sizes in nucleotides are indicated to the right of each blot. The membranes were re-probed for $5 \mathrm{~S}$ rRNA to control for equal loading of sRNAs (bottom). The signals in (B) were quantified, and values relative to the wt and normalized to 55 rRNA, are shown below each blot.

Tetrahymena thermophila and yeast [32,42]. Moreover, the tRNA half lengths are consistent with the known mechanistic action of RNases that cleave tRNA in the open loops, such as RNases T1, T2 and A [43].
A distinct fragmentation pattern was seen from tRNA Arg_cluster0. Sequencing identified two classes of Arg0tRFs, a $27 \mathrm{nt}$ long $5^{\prime}$ tRF and a 19-21 nt fragment mapping between the tRNA anticodon loop and the T-loop 

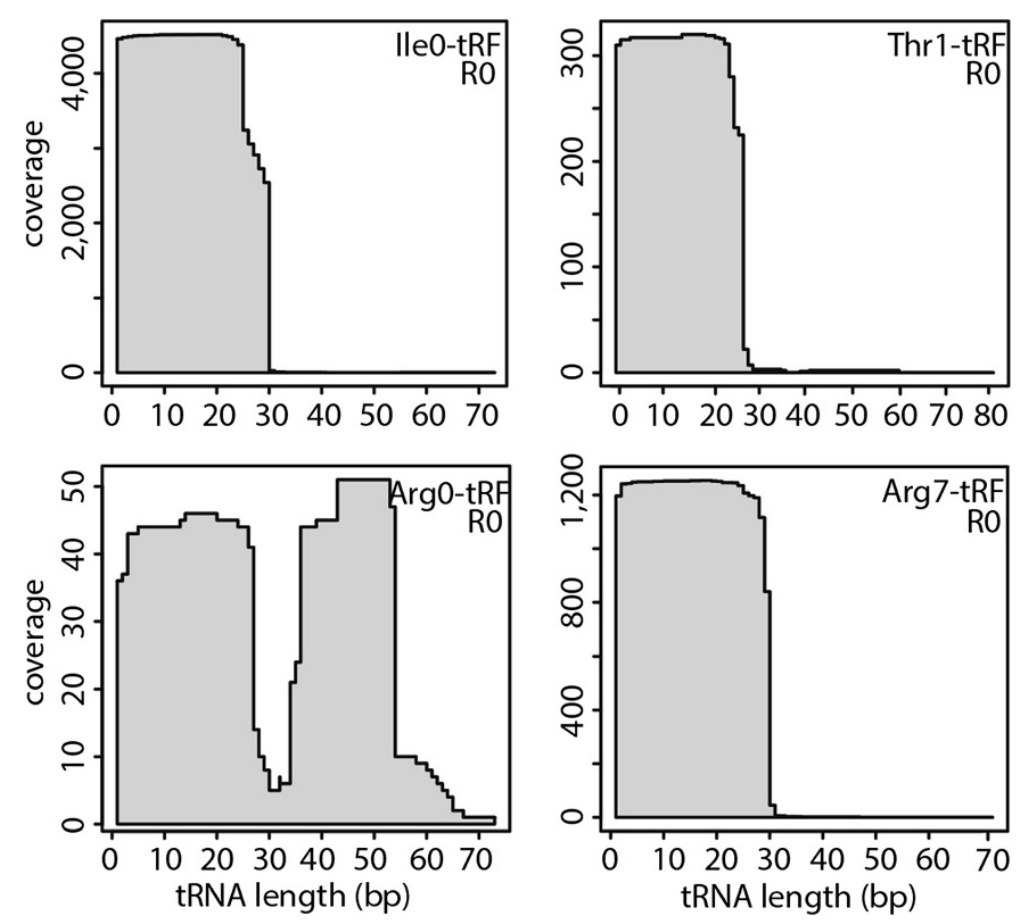

Figure 4 Sequence read coverage at tRNA clusters. sRNA read counts mapped along tRNA lle_cluster0, tRNA Thr_cluster1, tRNA Arg_cluster0 and Arg_cluster7 in isolate R0. The profile was very similar in isolate 3928A.

(Figure 4). Probing specifically for the internal fragment confirmed the presence of 19 and 21 nt tRFs (Figure 3) as well as detecting $38-45$ nt products (Additional file 4: Figure S3A). Similar-sized tRFs were detected using a probe designed to bind to the $3^{\prime}$ end of tRNA Arg_cluster0 (Additional file 4: Figure S3B). Sequenced Arg0 5' fragments were $27 \mathrm{nt}$ long, however tRNA half products of about 34 and $36 \mathrm{nt}$ were experimentally detected from the $5^{\prime}$ end (Additional file 4: Figure S3C). In conclusion, three types of tRFs appeared to be generated from tRNA Arg_cluster0: $5^{\prime}$ and $3^{\prime}$ half molecules through cleavage in the anticodon loop and an internal fragment generated by cleavages in both the anticodon- and T-loops (Figure 5).

\section{Detection of $3^{\prime}$ tRF sequences}

The majority of sequenced tRFs mapped to tRNA $5^{\prime}$ termini. This selective maintenance of one tRNA cleavage product over the other is analogous to the preferred cellular retention of siRNA and miRNA guide strands as opposed to their respective passenger and miRNA* strands. We next extended the analyses to include $3^{\prime}$ tRFs, performing Northern hybridizations on the same sporulating mycelium samples as the corresponding $5^{\prime}$ tRFs had been detected in. tRNA Ile_cluster0 was chosen as the first candidate, due to the ease of experimental detection of its $5^{\prime}$ tRFs. Despite the lack of reads from $3^{\prime}$ half Ile_cluster0 tRNAs in the SOLiD libraries and the Illumina mycelium library (Figure 4, Additional file 6: Figure S5), clear hybridization signals from $3^{\prime}$ tRFs were detectable (Additional file 4: Figure S3D). This finding was not a unique feature of this particular tRNA: no $3^{\prime}$ fragments were present from tRNA Arg_cluster7 in any of the sequencing libraries (Figure 4, Additional file 6: Figure S5) but fragments from both $5^{\prime}$ and $3^{\prime}$ tRNA halves were seen by Northern hybridization (Additional file 4: Figure S3E, F). This indicates that the cleavage products from both halves of the two tRNAs are maintained after anticodon loop processing, contradicting the results from sequencing.

\section{Changes in the tRF repertoire during host plant infection}

To examine tRNA fragmentation during $P$. infestans infection of potato, sRNA libraries were generated from potato leaves infected with isolate 88069 and a $D c l$-silenced transformant (PiDcl1), sampled at three time points: 24,48 and 72 hours post inoculation (hpi). These, and control samples from the mycelium life cycle stage, were sequenced using the Illumina HiSeq 2500 platform. Mapping of sRNA reads to the tRNA clusters revealed major length classes of 29, 31 and $33 \mathrm{nt}$, highly enriched for sense reads (Figure 1B). As in the SOLiD data, a bias was seen towards the tRNA $5^{\prime}$ end, although the over-representation was not as large (Tables 1 and 2, Additional file 7: Figure S6). The fraction of $5^{\prime}$ tRFs in both 88069 and the PiDcl1-silenced line was somewhat higher at the three infection 

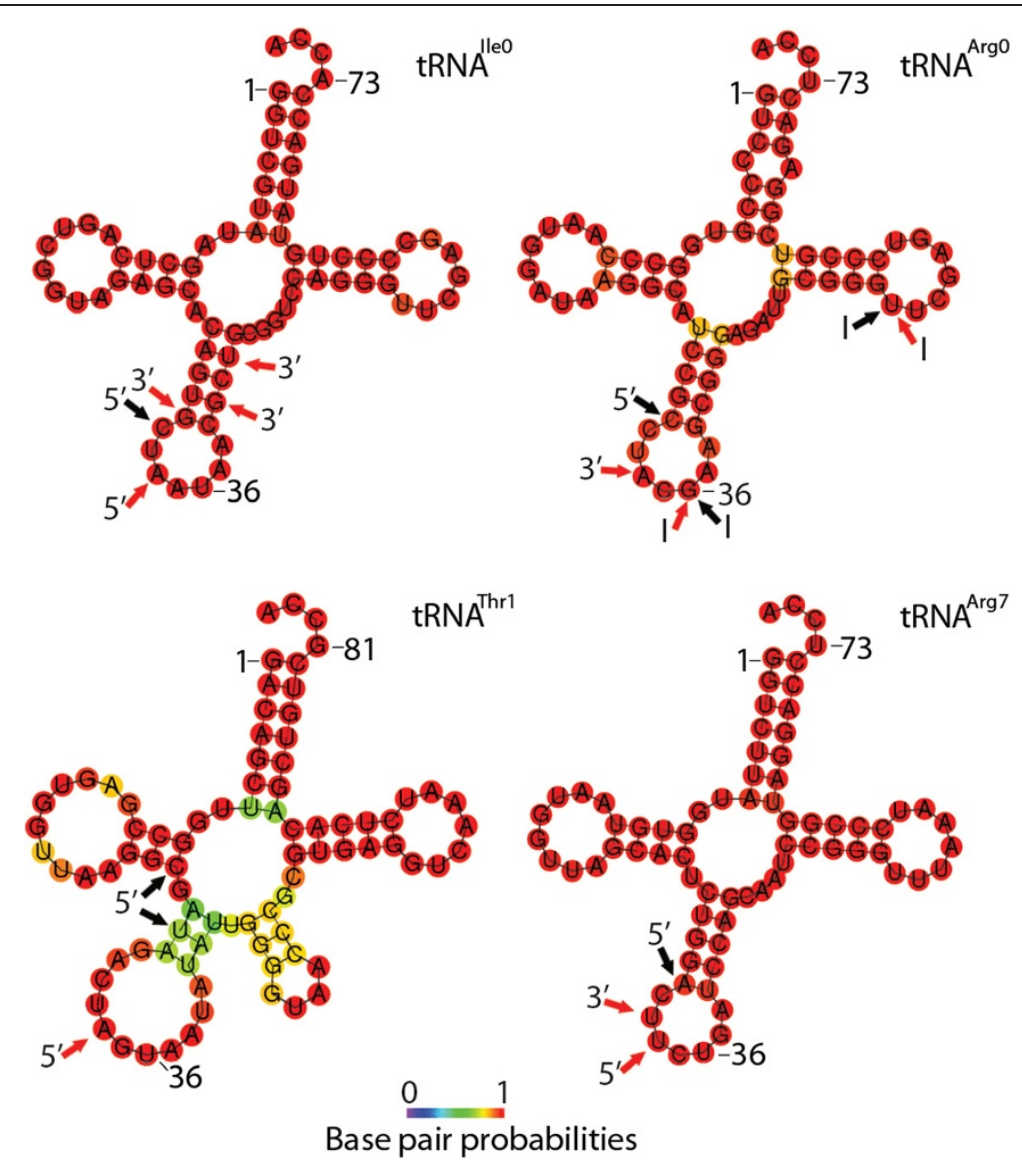

Figure 5 Putative tRNA structures and predicted cleavage sites. The predicted secondary structures of tRNA Ile_cluster0, tRNA Arg_clusterO, tRNA Thr_cluster 1 and tRNA Arg_cluster7 are shown. The color code depicts base pair probabilities. Black arrows show $5^{\prime}$ cleavage sites determined by sRNA sequencing while cleavage sites suggested from Northern hybridizations are shown by red arrows for $5^{\prime}$ tRFs, internal (I) tRFs or $3^{\prime}$ tRFs. The lengths of $3^{\prime}$ tRFs are calculated excluding the $3^{\prime}$ CCA.

time points (on average $71 \%$ and $79 \%$, respectively) than in mycelium (55\% and 49\%). A more dramatic change in the relative production of $5^{\prime}$ and $3^{\prime}$ tRNA halves was observed in T. cruzi, where under nutritional stress $98 \%$ of the tRFs derived from $5^{\prime}$ halves [34], while $87 \%$ were

Table 2 Distribution of tRFs mapping to the $5^{\prime}$ and $3^{\prime}$ halves of mature tRNA in 88069 and the PiDcl1 silenced mutant

\begin{tabular}{llllll}
\hline \multirow{2}{*}{ Library } & \multicolumn{3}{l}{ Number of reads } & (\%) \\
\cline { 2 - 5 } & $\mathbf{5}^{\prime}$ & $\mathbf{3}^{\prime}$ & Intermediate & Total & $\mathbf{5}^{\mathbf{\prime}^{\prime}}$ \\
\hline 88069 24 h & 9909 & 3740 & 698 & 14347 & 69 \\
$8806948 \mathrm{~h}$ & 13130 & 2932 & 1573 & 17635 & 74 \\
$8806972 \mathrm{~h}$ & 29752 & 8601 & 4067 & 42420 & 70 \\
88069 mycelia & 37540 & 22957 & 8300 & 68797 & 55 \\
PiDcl1 24 h & 5365 & 1059 & 443 & 6867 & 78 \\
PiDcl1 48 h & 8389 & 2032 & 680 & 11101 & 76 \\
PiDcl1 72 h & 3302 & 317 & 343 & 3962 & 83 \\
PiDcl1 mycelia & 75783 & 52973 & 25937 & 154693 & 49 \\
\hline
\end{tabular}

processed from $3^{\prime}$ halves in cells differentiated into the host-infective life cycle stage [44]. Differences in the proportions of $5^{\prime}$ mapping tRFs were observed between the isolates used in our study (90\% in R0, 83\% in 3928A and $55 \%$ in 88069 ). It should be noted that the lower proportion revealed from 88069 was from sequencing using Illumina technology while SOLiD was used for the R0 and 3928A isolates. It is therefore difficult to associate the observed differences in tRF abundance with any pathogenicity characteristics.

Despite the absence of sRNA sequencing reads derived from the $3^{\prime}$ ends of tRNA Ile_cluster 0 and tRNA Arg_cluster7, 3' tRFs were readily detected by Northern hybridization. This discrepancy might be due to sequencing bias, which is common in sRNA sequencing studies [45]. Factors that influence the process of cDNA construction, such as $5^{\prime}$ and $3^{\prime}$ nucleotide identities and modifications, in addition to sRNA secondary structure, could have biased our sequencing result towards $5^{\prime}$ tRFs. Similarly, different sequencing protocols vary in 
their sensitivity to the mentioned RNA features [45], making it difficult to do strict comparisons of data generated by different sequencing platforms. This could underlie the observed higher proportion of $5^{\prime}$ tRFs from the SOLiD-sequenced isolates (R0 and 3928A) compared to the Illumina-sequenced material (88069 and PiDcl1).

The Illumina sequencing data confirmed the $5^{\prime}$ tRNA halves observed by Northern hybridization from tRNA Ile_cluster0 and tRNA Arg_cluster7 as well as both the $5^{\prime}$ and the $3^{\prime}$ halves from tRNA Arg_cluster0. In contrast, only trace amounts of the 19 nt internal tRF from tRNA Arg_cluster0 were seen (Additional file 6: Figure S5 and Additional file 8: Figure S7). Cleavage products from Ile tRNA_cluster0 were abundant in the datasets from both sequencing platforms (Additional file 1: Table S1, Additional file 9: Table S2). The most prominent product from this tRNA was the $5^{\prime}$ tRNA half, as detected by both Northern hybridization and Illumina sequencing (Figure 3, Additional file 6: Figure S5). Notably, the sequence size distribution was very narrow at the mycelium stage, showing a sharp peak at $32 \mathrm{nt}$, while no single dominant size class was observable at 24 and 72 hpi (Additional file 8: Figure S7). Since half tRNA-sized Ile0-5' tRFs were the most mycelium-enriched of all tRFs (Additional file 9: Table S2 and Additional file 10: Figure S8), this suggests a reduction in the number of Ile_cluster0 tRNAs that are cleaved in the anticodon loop during infection.

\section{Differential tRF abundance during infection progression}

To compare the tRF read counts in-between the different time points and with the sample from cultured mycelium, heat maps were generated from the normalized read counts from each tRNA cluster. A number of tRFs in isolate 88069 showed elevated read numbers in one or more of the three infection-stage libraries. We chose to focus on the top twenty tRFs most abundant across the four libraries (Additional file 10: Figure S8). Both the second-most infection-responsive tRF (Pro1-tRF, 31 nt; Additional file 8: Figure S7) and the third-most infectionresponsive tRF (Leu6-tRF, $26 \mathrm{nt}$ ) peaked at the biotrophic stage of the interaction (24 hpi). This phase is characterized by plant mesophyll tissue invasion by $P$. infestans intercellular hyphae that acquire nutrients from the host [46]. The same two tRFs were shown to decrease at the transition stage (48 hpi) and decline further at necrotrophy (72 hpi). At this point the plant tissue was extensively colonized and sporulating lesions were formed on the leaf surface. Lys3-tRFs $(25,26 \mathrm{nt})$, the most elevated tRF under infection, peaked at this necrotrophic stage. A third tRF accumulation profile was seen for Cys1-tRF, Glu5-tRF, Asp0tRF and Gly1-tRF, which peaked at $48 \mathrm{hpi}$ (Additional file 10: Figure S8).
The first function ascribed to tRNA cleavage products was in regulation of various stress responses [47], a characteristic shared by organisms such as fungi (yeast), protists (Giardia, Tetrahymena), animals (mammals, flies) and plants (Arabidopsis) [14,32,42,48]. It is premature to infer a specific role for tRNA cleavage in the $P$. infestans-host interaction, but we note that: (1) a number of tRNA cleavage products accumulate at comparably higher levels during infection than in mycelium, and (2) tRFs accumulate differentially at the three infection time points. Possibly, some tRNAs or tRFs may be involved in the biotrophic-tonecrotrophic infection stage progression. Although further evidence is needed, it could be speculated that regulated tRNA fragmentation serves to decrease the levels of specific tRNAs, such as tRNA Pro_cluster1, for which the cleavage product was the most abundant tRF at 24 and 48 hpi. This mechanism could for example be part of a stressinduced translational re-programming response. It is notable that proline has been proposed to be an osmoregulator controlling zoospore discharge in P. nicotianae [49], and to regulate the osmotic pressure needed for penetration of the potato leaf cuticle in $P$. infestans [50]. The levels of free proline in the latter study were four times higher in the pre-infection life cycle stage compared to mycelium. The differential levels of Pro_cluster1 tRFs may reflect a mechanism used by $P$. infestans to regulate proline levels in the cell. The narrow size distribution of Pro1-tRFs and their accumulation pattern exclusively from the tRNA $5^{\prime}$ half, argue against the notion of Pro1-tRFs as random degradation products (Additional file 6: Figure S5, Additional file 8: Figure S7). Fragmentation of tRNA is typically not a feature of the well-characterized eukaryotic tRNA turnover pathways, which act from the tRNA $5^{\prime}$ and $3^{\prime}$ ends [39].

\section{Analysis of PiDcl and PiAgo involvement in tRF biogenesis}

It is being increasingly realized that tRFs associate with canonical RNAi components such as Dcl and Ago proteins in different organisms. In humans, studies of the interactions between hAgo3 and hAgo4 with 20-22 nt tRFs identified a tRF class formed through Dcl processing that is capable of trans-silencing of target sequences, similar to siRNAs and miRNAs [51]. In stressed Drosophila cells, tRNAs and tRFs compete with dsRNA for processing by the siRNA-specific Dcl (Dcr-2) [48]. Based on these previous studies and on the known, conserved roles of Dcl and Ago proteins in sRNA-binding and processing, we hypothesized that silencing PiDcl1 and the four PiAgos would lead to perturbed tRF levels in $P$. infestans. Constructs targeting PiDcl1, PiAgo1, PiAgo3, PiAgo4 and PiAgo5 were designed and stable hairpin-mediated gene silencing transformations were successful with all except PiAgo3. After analysis of the levels of transcript knockdown in the generated independent transformant lines by quantitative Reverse Transcription PCR (qRT- 
PCR), the most silenced line for each gene was chosen for further studies (Additional file 11: Figure S9). Silencing of PiDcl1 and PiAgos to similar levels has previously been shown to impact on sRNA accumulation and functionality of silencing $[20,21]$.

As seen by Northern blotting for nine different tRFs (Figure 3B, Additional file 5: Figure S4F-J), their production and stability was not negatively influenced by PiDcl1 knockdown, in contrast to findings in flies and mammals [48,52]. This was further confirmed by Illumina sequencing from PiDcl1, as no major differences in tRF levels or sizes were seen compared to the wild type. For illustration, the size distribution of Ile0-tRFs in 88069 and the PiDcl1-silenced line is shown in Additional file 8: Figure S7; similar results were found for other tRFs that were analyzed. We note that a role for PiDcl proteins in tRNA fragmentation cannot completely be ruled out, since a second Dcl protein (PiDcl2) was identified in the $P$. infestans genomic sequence trace archive after initiation of this study [22].

Reduced tRF levels were repeatedly observed in the PiAgo1-silenced line (e.g. Ile0-5' tRF, Trp2-5' tRF, Ser6$5^{\prime}$ tRF), and to a lesser extent in the PiAgo4-silenced line (Thr1-5'tRF, Arg0-tRF; Figure 3B). Fragments affected by PiAgo1 knockdown were not homogenous in size, reduced signals being observed for short tRFs (19 and $21 \mathrm{nt})$, as well as for 26-30 nt tRFs and for half-sized tRNA fragments. Levels of some $5^{\prime}$ halves were unperturbed in the PiAgo1-silenced line (tRNA Glu_cluster4, tRNA Leu_cluster0), suggesting that $P$. infestans has more than one tRNA fragmentation pathway. No decrease in tRF levels was seen in the PiAgo5-silenced line. This might be due to the low degree of gene silencing in this transformant (Additional file 11: Figure S9).

Ago proteins are specialized RNA binding proteins with preference for short RNAs such as miRNAs, siRNAs and piRNAs [53]. Crystallographic studies have shown that the PAZ domain accommodates the sRNA $3^{\prime}$ hydroxyl group, while the $5^{\prime}$ phosphate binding pocket resides within the MID domain. The structures and lengths of tRNAs and their cleavage products are distinct from those of canonical Ago-interacting sRNAs, making the formation of Ago-tRF complexes appear intriguing. Yet, new functions of Ago proteins continue to be discovered, and different Ago homologs vary in their ability to bind structured RNAs [53-55]. Couvillion and co-workers [56] proposed a model for the mechanism of Twi12-tRF complex formation in Tetrahymena, wherein stacking of the tRNA Tloop and acceptor stems creates a binding substrate of suitable size for Piwi recognition. Tight binding of the tRNA acceptor stem $3^{\prime}$ side would in this case make the $5^{\prime}$ endless protected from nucleases, explaining why $3^{\prime}$ tRFs are predominately loaded onto Twi12. It is possible that binding of PiAgol to tRNA similarly dictates the asymmetric accumulation of $P$. infestans tRFs preferentially from the tRNA $5^{\prime}$ side. The internal Arg0-tRF was also shown to be PiAgo1-dependent. This indicates that additional binding partners are involved in the putative PiAgo1-tRF complex, or that there are multiple PiAgo1tRF binding configurations.

\section{Crosstalk between RNA silencing and tRNA cleavage pathways}

At least two alternative roles for PiAgol in the tRNA cleavage pathways can be envisaged. On one hand, it is possible that PiAgo1 plays a direct role in tRF biogenesis, by endonuclease cleavage of tRNAs into $5^{\prime}$ and $3^{\prime}$ fragments. Alternatively, the protein might act at a step after tRNA cleavage, binding and stabilizing tRFs produced by another, as-yet unknown, nuclease. No convincing evidence for Ago-mediated tRNA cleavage has been put forward to date, thus the second possibility appears more likely. Besides Dcl and Ago proteins, the P. infestans genome encodes several additional classes of endoribonucleases. Two well-studied eukaryotic tRNA cleavage nucleases are yeast Rnylp (RNase T2 type) and mammalian Angiogenin (RNase A type) $[17,57,58]$. Since the RNase A superfamily is vertebrate-specific [59], we speculate that the $P$. infestans tRNA cleavage nuclease is of the RNase T2 class, of which at least five predicted proteins are encoded by the organism's genome (PITG_11433, PITG_16015, PITG_01495, PITG_15217 and PITG_08597). PiAgo1 is critical for maintaining gene silencing in P. infestans [20], which implies that bound tRFs have the potential to regulate RNA silencing through competition with other sRNAs for PiAgol loading. The tRNA cleavage products would then classify as "competing endogenous RNAs", regulatory RNAs competing for shared molecular targets and regulating each other through RNA-RNA crosstalk [60].

\section{Conclusions}

Deep sequencing is a powerful tool for sRNA research. Biases inherent in protocols and chemistries used in different platforms are however inevitable [45]. With this in mind, we conclude that this study identified a number of tRFs in the stramenopile P. infestans. By sRNA sequencing and Northern blot analysis in three isolates, we show that tRFs accumulate from both $5^{\prime}$ and $3^{\prime}$ halves of mature tRNA. According to sequencing data, $5^{\prime}$ tRFs were more abundant than $3^{\prime}$ tRFs under both asexual development and infection of host tissue, whereas the relative proportion of $5^{\prime}$ fragments was elevated during host infection. Overall, a number of tRFs were identified that seem more abundant during plant infection, possibly regulating pathogenicity related functions, analogous to recent findings of sRNAs as key players in host-pathogen interactions. Finally, knockdown of the genes encoding $P$. infestans Dcl and Ago proteins suggested an involvement 
of PiAgol in the tRF pathways. This crosstalk with $P$. infestans canonical RNA silencing pathways might lead to competition with other classes of sRNAs for PiAgol binding, and suggests a mechanism through which tRFs could regulate the activities of the RNA silencing machinery.

\section{Methods}

\section{$P$. infestans culturing and transformation}

sRNA preparations from $P$. infestans wild type isolates R0, 3928A and 88069 and from a PiDcl1-silenced transformant were used for deep sequencing. Culturing conditions, preparation of life cycle stage samples [21], infections and leaf sample collection (88069 and PiDcl1, potato cultivar Desirée) were as previously described [20]. The methods for designing inverted repeat silencing constructs, generation of stable $P$. infestans transformants and their maintenance were as for earlier reported transformants [21]. Primers used for cloning from $P$. infestans DNA are listed in the same study.

\section{Quantitative reverse transcription PCR analysis}

Extraction of total RNA, cDNA synthesis, qRT-PCR, and subsequent data analysis were carried out as outlined by Vetukuri et al. [20] including primer sequences. Transcript levels were normalized to the internal control PiActA (AAA33749) and presented as the fold change relative to the calibrator sample (88069 mycelium).

\section{sRNA sequencing and computational analysis}

Total RNA was extracted using the mirVana ${ }^{\text {Tix }}$ miRNA isolation Kit (Ambion). For SOLiD sequencing, the methods for RNA library preparation, deep sequencing and data analysis were as reported [21], except that the sRNA read mapping was done with increased stringency (read length 19-30 nt). Illumina library preparation was done with the Illumina TruSeq small RNA sample preparation kit and the sequencing reactions were run on a HiSeq 2500 platform at SciLifeLab (Stockholm, Sweden). All Illumina adaptor molecules were first filtered out from the sRNA sequences, and reads shorter than 18 bases or having less than 5 bp adaptor sequence were excluded from further analysis. The sRNA reads were aligned to the P. infestans genome tRNA dataset (http:// www.broadinstitute.org) using bowtie2 v2.1.0 [61] by first clustering the tRNA sequences with cd-hit [31], using $90 \%$ identity as a threshold for assignment to a cluster. From the clusters, a consensus sequence was constructed using the most common base at each position. Recording of sRNA lengths, numbers, starting base and sense/antisense reads was done using SAMtools v0.1.19 [62] and custom python scripts. The counts of reads mapping to each of the tRNA clusters were obtained using the BEDTools intersect command [63], and normalized using the R package DESeq [64]. Heatplots were generated from the normalized read counts at each tRNA cluster using the gplots package (cran.r-project.org/web/packages/gplots/) in the statistical software package R v3.0.2. Unless otherwise stated, all calculations were performed in R (http:// www.R-project.org) [65].

\section{tRNA secondary structure prediction}

The Vienna RNAfold webserver was used for prediction of tRNA cloverleaf secondary structures (http://rna.tbi. univie.ac.at) [41]. Default settings were used, except for changing the folding temperature to $20^{\circ} \mathrm{C}$ to reflect the optimal growth temperature of $P$. infestans and not allowing the CCA end to form any base pairs.

\section{sRNA Northern hybridization}

Low-molecular-weight fraction RNA was extracted from sporulating mycelium and analyzed by Northern hybridization [21] using DNA oligonucleotides $5^{\prime}$ endlabeled with $\gamma_{-}{ }^{32}$ P-ATP (Additional file 12: Table S3). A probe complementary to $P$. infestans $5 \mathrm{~S}$ rRNA was used for the loading control. Quantification of bands was done with the program QuantityOne (BioRad) and normalized to the loading control.

\section{Availability of supporting data}

The SOLiD sequencing data sets supporting the results of this article are available in NCBI's Gene Expression Omnibus (GEO) repository, accession GSE62674 (http:// www.ncbi.nlm.nih.gov/geo/query/acc.cgi?acc=GSE62674). The Illumina sequencing data sets are available in the GEO database, accession GSE63292 (http://www.ncbi.nlm. nih.gov/geo/query/acc.cgi?acc=GSE63292).

\section{Additional files}

Additional file 1: Table S1. SOLiD sRNA sequencing reads mapped to tRNA clusters in P. infestans isolates R0 and 3928A. Four life cycle stage samples were analyzed from each isolate.

Additional file 2: Figure S1. Proportion of reads mapping to either the $5^{\prime}$ or the $3^{\prime}$ end of tRNA (see labels at right). Four sequenced life cycle stages in RO.

Additional file 3: Figure S2. Proportion of reads mapping to either the $5^{\prime}$ or the $3^{\prime}$ end of tRNA (see labels at right). Four sequenced life cycle stages in 3928A.

Additional file 4: Figure S3. Detection of $5^{\prime}$ and $3^{\prime}$ half tRNAs. Northern hybridization in wild type isolates and the PiDcll silenced line. (A, B) $3^{\prime}$ half tRNAs from tRNA Arg_clusterO. (C) $5^{\prime}$ half tRNAs from tRNA Arg_clusterO. (D) $3^{\prime}$ half tRNAs from tRNA lle_clusterO. (E) $5^{\prime}$ half tRNAs from tRNA Arg_cluster7. (F) $3^{\prime}$ half tRNAs from tRNA Arg_cluster7. Approximate sizes in nucleotides are indicated to the right of each blot. Shown below each tRF Northern blot is the same membrane re-probed for $5 S$ rRNA to control for equal loading. The signals in (A) were quantified and the value in PiDCl1 is shown to the right of the blot, relative to the wild type (wt) 88069 and normalized to 5S rRNA.

Additional file 5: Figure S4. Detection of $5^{\prime}$ half tRNAs in wild type isolates and the PiDcl1 silenced line. Northern hybridization in (A-E) isolates R0 and 3928A and (F-J) isolate 88069 and the PiDCll silenced line. (A) tRNA Met_cluster3. (B) tRNA Asn_cluster1. (C) tRNA Asp_clustero. 
(D) tRNA Arg_cluster4. (E) tRNA Gly_cluster0. (F) tRNA Glu_cluster4. (G) tRNA Arg_cluster7. (H) tRNA Leu_cluster0. (I) tRNA Trp_cluster2. (J) tRNA Ser_cluster6. Approximate sizes in nucleotides are indicated to the right of each blot. Shown below each tRF Northern blot is the same membrane re-probed for $5 \mathrm{~S}$ rRNA to control for equal loading of SRNAs. The signals in (F-J) were quantified and values in PiDcll are shown to the right of the blot, relative to the wild type (wt) 88069 and normalized to 5 S rRNA.

Additional file 6: Figure S5. Sequence read coverage at tRNA clusters. sRNA read counts mapped along tRNA lle_cluster0, tRNA Arg_cluster7, tRNA Arg_cluster0, and tRNA Pro_cluster1 in the mycelium life cycle stage library and the three infection stage libraries in isolate 88069. The $y$-axis in each graph represents the total tRF read count.

Additional file 7: Figure S6. Proportion of reads mapping to either the $5^{\prime}$ or the $3^{\prime}$ end of tRNA (see labels at right). The mycelium life cycle stage (myc) and three infection stage time points (24, 48 and 72 hpi) in isolate 88069 .

Additional file 8: Figure S7. Size distribution of Illumina sRNA reads mapping to individual tRNA clusters. SRNA sequence read lengths in the three infection stage libraries and the mycelium life cycle stage library in isolate 88069 and in the PiDcll silenced mutant. Y-axis: the percentage mapped sRNA reads of each length class.

Additional file 9: Table S2. Illumina sRNA sequencing reads mapped to tRNA clusters in isolate 88069 and the PiDcl 1 silenced transformant. Three infection stage samples (24, 48 and $72 \mathrm{hpi}$ ) and one mycelium stage sample were analyzed from each line.

Additional file 10: Figure S8. Heatmap displaying tRNA-mapping read numbers. The top 20 tRFs exhibiting the highest numbers of normalized read counts across the four sequenced libraries in isolate 88069. 24, 48 and 72 hpi; three infection time points, myc; mycelium. Color key: normalized read counts.

Additional file 11: Figure S9. Transcript levels in hairpin-transformed lines assayed by real-time RT-PCR. The four silenced lines used for Northern blot analysis of tRF levels. mRNA levels were normalized to the ActinA (PiActA) reference gene and are presented relative to the wild type isolate 88069 (assigned a value of 1.0). Total numbers of individual lines analyzed per gene: PiDcl1- 10, PiAgo 1- 8, PiAgo4- 10 and PiAgo5- 6. Error bars represent confidence intervals from two technical replicates per sample in the PCR reaction.

Additional file 12: Table S3: Sequences of oligonucleotides used as DNA probes.

\section{Abbreviations}

Ago: Argonaute; Dcl: Dicer-like; hpi: Hours post inoculation; sRNA: Small RNA; tRF: tRNA-derived RNA fragment

\section{Competing interests}

The authors declare that they have no competing interests.

\section{Authors' contributions}

AKMÅ, RRV and CD conceived and designed the experiments. AKMÅ, RRV and SNJ performed the experiments. SNJ, AOA and SCW contributed to the analysis and interpretation of the data. JF and PC performed the bioinformatics analyses. AKMÅ, RRV, SCW and CD wrote the manuscript. All authors read and approved the manuscript.

\section{Acknowledgements}

The authors are grateful for support from Science for Life (SciLife) Laboratory, the National Genomics Infrastructure (NGI), Sweden, the Knut and Alice Wallenberg Foundation and UPPMAX for providing assistance in massively parallel sequencing and computational infrastructure. This work was supported by Formas, VR, Helge Ax:son Johnsons Foundation and SLU. SCW and AOA were supported by grants from the Scottish Government Rural and Environment Science and Analytical Services Division (RESAS), and the Biotechnology and Biological Sciences Research Council (BBSRC). The funders had no role in study design, data collection and analysis, decision to publish, or preparation of the manuscript.

\section{Author details}

'Department of Plant Biology, Uppsala BioCenter, Linnéan Centre for Plant Biology, Swedish University of Agricultural Sciences, PO. Box 7080, SE-75007
Uppsala, Sweden. ${ }^{2}$ Cell and Molecular Sciences, The James Hutton Institute, Invergowrie, Dundee DD2 5DA, UK. ${ }^{3}$ Current affiliation: Department of Evolutionary Biology, Uppsala University, SE-75236 Uppsala, Sweden.

Received: 8 September 2014 Accepted: 24 November 2014 Published online: 10 December 2014

\section{References}

1. Beakes GW, Glockling SL, Sekimoto S: The evolutionary phylogeny of the oomycete "fungi". Protoplasma 2012, 249(1):3-19.

2. Dick MW: The Peronosporomycetes. In The Mycota VII. Systematics and Evolution Part A. Edited by McLaughlin DJ, McLaughlin EG, Lemke PA. Berlin Heidelberg New York: Springer; 2001:39-72.

3. Adl SM, Simpson AG, Lane CE, Lukeš J, Bass D, Bowser SS, Brown MW, Burki F, Dunthorn M, Hampl V, Heiss A, Hoppenrath M, Lara E, Le Gall L, Lynn DH, McManus H, Mitchell EA, Mozley-Stanridge SE, Parfrey LW, Pawlowski J, Rueckert S, Shadwick RS, Schoch CL, Smirnov A, Spiegel FW: The revised classification of eukaryotes. J Eukaryot Microbiol 2012, 59(5):429-493.

4. Lamour KH, Win J, Kamoun S: Oomycete genomics: new insights and future directions. FEMS Microbiol Lett 2007, 274(1):1-8.

5. Phillips AJ, Anderson VL, Robertson EJ, Secombes CJ, van West P: New insights into animal pathogenic oomycetes. Trends Microbiol 2008, 16(1):13-19

6. Fry W: Phytophthora infestans: the plant (and $R$ gene) destroyer. Mol Plant Pathol 2008, 9(3):385-402.

7. Haas BJ, Kamoun S, Zody MC, Jiang RH, Handsaker RE, Cano LM, Grabherr M, Kodira CD, Raffaele S, Torto-Alalibo T, Bozkurt TO, Ah-Fong AM, Alvarado L, Anderson VL, Armstrong MR, Avrova A, Baxter L, Beynon J, Boevink PC, Bollmann SR, Bos Jl, Bulone V, Cai G, Cakir C, Carrington JC, Chawner M, Conti L, Costanzo S, Ewan R, Fahlgren N, et al: Genome sequence and analysis of the Irish potato famine pathogen Phytophthora infestans. Nature 2009, 461(7262):393-398.

8. Gijzen M: Runaway repeats force expansion of the Phytophthora infestans genome. Genome Biol 2009, 10(10):241.

9. Raffaele S, Win J, Cano LM, Kamoun S: Analyses of genome architecture and gene expression reveal novel candidate virulence factors in the secretome of Phytophthora infestans. BMC Genomics 2010, 11:637.

10. Pais M, Win J, Yoshida K, Etherington GJ, Cano LM, Raffaele S, Banfield MJ, Jones A, Kamoun S, Saunders DGO: From pathogen genomes to host plant processes: the power of plant parasitic oomycetes. Genome Biol 2013, 14(6):211.

11. Ghildiyal M, Zamore PD: Small silencing RNAs: an expanding universe. Nat Rev Genet 2009, 10(2):94-108.

12. Claycomb JM: Ancient endo-siRNA pathways reveal new tricks. Curr Biol 2014, 24(15):R703-R715.

13. Czech B, Hannon GJ: Small RNA sorting: matchmaking for Argonautes. Nat Rev Genet 2011, 12(1):19-31.

14. Li Y, Luo J, Zhou H, Liao JY, Ma LM, Chen YQ, Qu LH: Stress-induced tRNA-derived RNAs: a novel class of small RNAs in the primitive eukaryote Giardia lamblia. Nucleic Acids Res 2008, 36(19):6048-6055.

15. Lee YS, Shibata Y, Malhotra A, Dutta A: A novel class of small RNAs: tRNA-derived RNA fragments (tRFs). Genes Dev 2009, 23(22):2639-2649.

16. Sobala A, Hutvagner G: Transfer RNA-derived fragments: origins, processing and functions. Wiley Interdiscip Rev RNA 2011, 2(6):853-862.

17. Gebetsberger J, Polacek N: Slicing tRNAs to boost functional ncRNA diversity. RNA Biol 2013, 10(12):1798-1806.

18. van West P, Kamoun S, van 't Klooster JW, Govers F: Internuclear gene silencing in Phytophthora infestans. Mol Cell 1999, 3(3):339-348.

19. Ah-Fong AM, Bormann-Chung CA, Judelson HS: Optimization of transgenemediated silencing in Phytophthora infestans and its association with small-interfering RNAs. Fungal Genet Biol 2008, 45(8):1197-1205.

20. Vetukuri RR, Avrova AO, Grenville-Briggs LJ, Van West P, Söderbom F, Savenkov El, Whisson SC, Dixelius C: Evidence for involvement of Dicer-like, Argonaute and histone deacetylase proteins in gene silencing in Phytophthora infestans. Mol Plant Pathol 2011, 12(8):772-785.

21. Vetukuri RR, Åsman AK, Tellgren-Roth C, Jahan SN, Reimegård J, Fogelqvist J, Savenkov E, Söderbom F, Avrova AO, Whisson SC, Dixelius C: Evidence for small RNAs homologous to effector-encoding genes and transposable elements in the oomycete Phytophthora infestans. PLoS One 2012, 7(12):e51399.

22. Fahlgren N, Bollmann SR, Kasschau KD, Cuperus JT, Press CM, Sullivan CM, Chapman EJ, Hoyer JS, Gilbert KB, Grünwald NJ, Carrington JC: 
Phytophthora have distinct endogenous small RNA populations that include short interfering and microRNAs. PLOS One 2013, 8(10):e77181.

23. Whisson SC, Avrova AO, van West P, Jones JT: A method for doublestranded RNA-mediated transient gene silencing in Phytophthora infestans. Mol Plant Pathol 2005, 6(2):153-163.

24. Judelson HS, Tani S: Transgene-induced silencing of the zoosporogenesisspecific NIFC gene cluster of Phytophthora infestans involves chromatin alterations. Eukaryot Cell 2007, 6(7):1200-1209.

25. van West P, Shepherd SJ, Walker CA, Li S, Appiah AA, Grenville-Briggs LJ, Govers F, Gow NA: Internuclear gene silencing in Phytophthora infestans is established through chromatin remodelling. Microbiology 2008, 154(5):1482-1490.

26. Vetukuri RR, Tian Z, Avrova AO, Savenkov El, Dixelius C, Whisson SC: Silencing of the PiAvr3a effector-encoding gene from Phytophthora infestans by transcriptional fusion to a short interspersed element. Fungal Biol 2011, 115(12):1225-1233.

27. Vetukuri RR, Åsman AK, Jahan SN, Avrova AO, Whisson SC, Dixelius C: Phenotypic diversification by gene silencing in Phytophthora plant pathogens. Commun Integr Biol 2013, 6(6):e25890.

28. Weiberg A, Wang M, Lin FM, Zhao H, Zhang Z, Kaloshian I, Huang HD, Jin H: Fungal small RNAs suppress plant immunity by hijacking host RNA interference pathways. Science 2013, 342(6154):118-123.

29. Flier WG, Turkensteen $L$, van den Bosch GBM, Vereijken PFG, Mulder A: Differential interaction of Phytophthora infestans on tubers of potato cultivars with different levels of blight resistance. Plant Pathol 2001, 50:292-301.

30. Cooke DE, Cano LM, Raffaele S, Bain RA, Cooke LR, Etherington GJ, Deahl KL, Farrer RA, Gilroy EM, Goss EM, Grünwald NJ, Hein I, MacLean D, McNicol JW, Randall E, Oliva RF, Pel MA, Shaw DS, Squires JN, Taylor MC, Vleeshouwers VG, Birch PR, Lees AK, Kamoun S: Genome analyses of an aggressive and invasive lineage of the Irish potato famine pathogen. PLoS Pathog 2012, 8(10):e1002940.

31. Li W, Godzik A: Cd-hit: a fast program for clustering and comparing large sets of protein or nucleotide sequences. Bioinformatics 2006 22(13):1658-1659.

32. Lee SR, Collins K: Starvation-induced cleavage of the tRNA anticodon loop in Tetrahymena thermophila. J Biol Chem 2005, 280(52):42744-42749.

33. Hsieh LC, Lin SI, Shih AC, Chen JW, Lin WY, Tseng CY, Li WH, Chiou TJ: Uncovering small RNA-mediated responses to phosphate deficiency in Arabidopsis by deep sequencing. Plant Physiol 2009, 151(4):2120-2132.

34. Garcia-Silva MR, Frugier M, Tosar JP, Correa-Dominguez A, Ronalte-Alves L, Parodi-Talice A, Rovira C, Robello C, Goldenberg S, Cayota A: A population of tRNA-derived small RNAs is actively produced in Trypanosoma cruzi and recruited to specific cytoplasmic granules. Mol Biochem Parasitol 2010, 171(2):64-73.

35. Franzén $O$, Arner E, Ferella M, Nilsson D, Respuela P, Carninci P, Hayashizak $Y$, Åslund L, Andersson B, Daub CO: The short non-coding transcriptome of the protozoan parasite Trypanosoma cruzi. PLoS Negl Trop Dis 2011, 5(8):e1283.

36. Tripathy S, Tyler BM: The repertoire of transfer RNA genes is tuned to codon usage bias in the genomes of Phytophthora sojae and Phytophthora ramorum. Mol Plant Microbe Interact 2006, 19(12):1322-1328.

37. Mi S, Cai T, Hu Y, Chen Y, Hodges E, Ni F, Wu L, Li S, Zhou H, Long C, Chen S, Hannon GJ, Qi Y: Sorting of small RNAs into Arabidopsis Argonaute complexes is directed by the $5^{\prime}$ terminal nucleotide. Cell 2008, 133(1):116-127.

38. Kirsebom LA: RNase P RNA mediated cleavage: substrate recognition and catalysis. Biochimie 2007, 89(10):1183-1194.

39. Phizicky EM, Hopper AK: tRNA biology charges to the front. Genes Dev 2010, 24(17):1832-1860.

40. Dupasquier M, Kim S, Halkidis $K$, Gamper $H$, Hou YM: tRNA integrity is a prerequisite for rapid CCA addition: implication for quality control. $J \mathrm{Mol}$ Biol 2008, 379(3):579-588.

41. Gruber AR, Lorenz R, Bernhart SH, Neuböck R, Hofacker IL: The Vienna RNA websuite. Nucleic Acids Res 2008, 36(Web Server issue):W70-W74.

42. Thompson DM, Lu C, Green PJ, Parker R: tRNA cleavage is a conserved response to oxidative stress in eukaryotes. RNA 2008, 14(10):2095-2103.

43. Durdevic Z, Schaefer M: tRNA modifications: necessary for correct tRNA-derived fragments during the recovery from stress? Bioessays 2013, 35(4):323-327.
44. Reifur L, Garcia-Silva MR, Poubel SB, Alves LR, Arauco P, Buiar DK, Goldenberg S, Cayota A, Dallagiovanna B: Distinct subcellular localization of tRNA-derived fragments in the infective metacyclic forms of Trypanosoma cruzi. Mem Inst Oswaldo Cruz 2012, 107(6):816-819.

45. Raabe CA, Tang TH, Brosius J, Rozhdestvensky TS: Biases in small RNA deep sequencing data. Nucleic Acids Res 2014, 42(3):1414-1426.

46. Avrova AO, Boevink PC, Young V, Grenville-Briggs LJ, van West P, Birch PR, Whisson SC: A novel Phytophthora infestans haustorium-specific membrane protein is required for infection of potato. Cell Microbiol 2008, 10(11):2271-2284.

47. Garcia-Silva MR, Cabrera-Cabrera F, Güida MC, Cayota A: Hints of tRNA-derived small RNAs role in RNA silencing mechanisms. Genes 2012, 3(4):603-614.

48. Durdevic Z, Mobin MB, Hanna K, Lyko F, Schaefer M: The RNA methyltransferase Dnmt2 is required for efficient Dicer-2-dependent siRNA pathway activity in Drosophila. Cell Rep 2013, 4(5):931-937.

49. Ambikapathy J, Marshall JS, Hocart CH, Hardham AR: The role of proline in osmoregulation in Phytophthora nicotianae. Fungal Genet Biol 2002, 35(3):287-299.

50. Grenville-Briggs $\sqcup$, Avrova AO, Bruce CR, Williams A, Whisson SC, Birch PR, van West P: Elevated amino acid biosynthesis in Phytophthora infestans during appressorium formation and potato infection. Fungal Genet Biol 2005, 42(3):244-256.

51. Haussecker D, Huang Y, Lau A, Parameswaran P, Fire AZ, Kay MA: Human tRNA-derived small RNAs in the global regulation of RNA silencing. RNA 2010, 16(4):673-695.

52. Cole C, Sobala A, Lu C, Thatcher SR, Bowman A, Brown JW, Green PJ, Barton GJ, Hutvagner G: Filtering of deep sequencing data reveals the existence of abundant Dicer-dependent small RNAs derived from tRNAs. RNA 2009, 15(12):2147-2160.

53. Meister G: Argonaute proteins: functional insights and emerging roles. Nat Rev Genet 2013, 14(7):447-459.

54. Huang V, Li LC: Demystifying the nuclear function of Argonaute proteins. RNA Biol 2014, 11(1):18-24.

55. Qu F, Ye X, Morris TJ: Arabidopsis DRB4, AGO1, AGO7, and RDR6 participate in a DCL4-initiated antiviral RNA silencing pathway negatively regulated by DCL1. Proc Natl Acad Sci U S A 2008, 105(38):14732-14737.

56. Couvillion MT, Sachidanandam R, Collins K: A growth-essential Tetrahymena Piwi protein carries tRNA fragment cargo. Genes Dev 2010, 24(24):2742-2747.

57. Fu H, Feng J, Liu Q, Sun F, Tie Y, Zhu J, Xing R, Sun Z, Zheng X: Stress induces tRNA cleavage by angiogenin in mammalian cells. FEBS Lett 2009, 583(2):437-442.

58. Thompson DM, Parker R: The RNase Rny1p cleaves tRNAs and promotes cell death during oxidative stress in Saccharomyces cerevisiae. J Cell Biol 2009, 185(1):43-50.

59. Hillwig MS, Rizhsky L, Wang Y, Umanskaya A, Essner JJ, Maclntosh GC: Zebrafish RNase T2 genes and the evolution of secretory ribonucleases in animals. BMC Evol Biol 2009, 9:170.

60. Tay Y, Rinn J, Pandolfi PP: The multilayered complexity of ceRNA crosstalk and competition. Nature 2014, 505(7483):344-352.

61. Langmead B, Salzberg SL: Fast gapped-read alignment with Bowtie 2. Nat Methods 2012, 9(4):357-359.

62. Li H, Handsaker B, Wysoker A, Fennell T, Ruan J, Homer N, Marth G, Abecasis G, Durbin R, 1000 Genome Project Data Processing Subgroup: The sequence Alignment/Map format and SAMtools. Bioinformatics 2009 25(16):2078-2079.

63. Quinlan AR, Hall IM: BEDTools: a flexible suite of utilities for comparing genomic features. Bioinformatics 2010, 26(6):841-842.

64. Anders S, Huber W: Differential expression analysis for sequence count data. Genome Biol 2010, 11(10):R106.

65. R Development Core Team: R: A Language and Environment for Statistical Computing. Vienna, Austria: R Foundation for Statistical Computing; 2008. http://www.R-project.org. ISBN 3-900051-07-0.

doi:10.1186/s12866-014-0308-1

Cite this article as: Åsman et al.: Fragmentation of tRNA in Phytophthora infestans asexual life cycle stages and during host plant infection. BMC Microbiology 2014 14:308. 